\title{
Essai d'analyse écologique de l'espace montagnard
}

\section{Georges Bertrand, Olivier Dollfus}

\section{Résumé}

Les espaces naturels montagnards correspondent, malgré leur diversité, à un même schéma d'organisation écologique. Afin d'élaborer une première esquisse de « modèle » d'espace montagnard, on a réuni et classé selon l'échelle spatiale les « effets » écologiques qui commandent la distribution et la dynamique des paysages montagnards, et qui pourraient bien être des « lois » écologiques encore incomplètement formulées.

\begin{abstract}
An attempt at ecological analysis in mountainous areas. - In spite of their diversity, naturally mountainous areas conform to a single scheme of ecological organization. To the end of elaborating a preliminary "model" for mountainous areas, ecological "effects" have been brought together and classed according to their areal scale. These effects dominate the distribution and development of mountainous landscapes and could well represent ecological "laws" as yet only partially formulated.
\end{abstract}

Citer ce document / Cite this document :

Bertrand Georges, Dollfus Olivier. Essai d'analyse écologique de l'espace montagnard. In: Espace géographique, tome 2, ${ }^{\circ} 3$, 1973. pp. 165-170;

doi : https://doi.org/10.3406/spgeo.1973.1397

https://www.persee.fr/doc/spgeo_0046-2497_1973_num_2_3_1397

Fichier pdf généré le 02/04/2018 


\section{ESSAI D'ANALYSE ÉCOLOGIQUE DE L'ESPACE MONTAGNARD}

\author{
Georges BERTRAND \\ CIMA-ERA 427 (C.N.R.S.) \\ Université de Toulouse-Le Mirail
}

\author{
Olivier DOLLFUS \\ Laboratoire de Géographie physique \\ Université de Paris VII
}

RESUME. Les espaces naturels montagnards correspondent, malgré leur diversité, à un même schéma d'organisation écologique. Afin d'élaborer une première esquisse de «modèle» d'espace montagnard, on a réuni et classé selon l'échelle spatiale les «effets» écologiques qui commandent la distribution et la dynamique des paysages montagnards, et qui pourraient bien être des «lois» écologiques encore incomplètement formulées.

ABSTRACT. An attempt at ecological analysis in mountainous areas. - In spite of their diversity, naturally mountainous areas conform to a single scheme of ecological organization. To the end of elaborating a preliminary "model" for mountainous areas, ecological "effects" have been brought together and classed according to their areal scale. These effects dominate the distribution and development of mountainous landscapes and could well represent ecological "laws" as yet only partially formulated.

Comme tous les objets géographiques ou écologiques complexes (ville, campagne, forêt, rivière), la montagne est une réalité quotidienne de la vie et du langage, une sorte de combinaison compréhensive et directement ressentie, qui appartient à tous et que chacun croit comprendre et dominer. Toutefois, cette banalité qui remplit les yeux borne l'esprit d'analyse. Il suffit de considérer les définitions que l'on donne de la montagne. Elles frappent par leur indigence. La plus commune et la moins insatisfaisante d'entre elles retient ce fait universel et fondamental que constitue l'étagement des milieux commandé par le gradient thermique altitudinal. Cela revient à isoler du complexe, donc à privilégier, un seul critère écologique, aussi important soit-il, c'est-à-dire à accorder une importance démesurée à un système taxonomique partiel, acceptable seulement pour une échelle spatiale déterminée (les étages de végétation). La prise en considération de la zonalité aurait pu enrichir ce schéma si on n'en était resté à un niveau élémentaire de discussion (la montagne est-elle un milieu «zonal» ou «azonal»?). Rares sont les auteurs, comme C. Troll et G. Rougerie, qui sont allés plus avant, et qui ont essayé de reconnaitre une spécificité écologique et une organisation interne propres aux milieux montagnards (1).

Certes, tous les grands phénomènes montagnards «naturels» sont généralement bien connus dans leurs catégories respectives. Dans l'état actuel de la recherche en géographie physique et en écologie, on peut, soit fixer l'image individuelle, monographique, d'un massif montagneux, soit conduire l'analyse séparée d'un élément du milieu montagnard ou d'un sousensemble (modelés, étages de végétation). Par contre, le milieu montagnard en tant que tel reste rebelle à l'analyse globale, à la généralisation théorique et. à la «modélisation ». Mais, paradoxalement, on reconnaît communément qu'en dépit de la diversité de leurs situations géographiques et de la variété de leurs paysages, toutes les montagnes du monde relèvent d'une même entité géographique...

Cette contradiction formelle bloque l'analyse $\mathrm{du}$ fait montagnard. Elle rend les comparaisons entre les montagnes très superficielles et approximatives, interdit les classifications systématiques et complique

(1) Voir l'introduction de la Biogéographie des montagnes de G. Rougerie. Paris, C.D.U., p. 14. 
l'étude des mécanismes spécifiques. Les références à des principes d'organisation de l'espace montagnard tels que l'étagement bioclimatique, le rôle de l'exposition, etc., ne sont pas rares, mais elles sont utilisées en ordre dispersé sans élaborer un schéma systématique. Your saisir le plus complètement et le plus rapidement possible l'organisation de l'espace «naturel» himalayen, on a d'abord eu recours à la connaissance directe que l'on pouvait avoir d'autres montagnes (Andes, Alpes, Pyrénées, montagnes ibériques, etc.). Mais ces comparaisons occasionnelles d'individu à individu se sont révélées artificielles, dangereuses, inefficaces. D'où l'obligation de fabriquer un outil. En l'occurence, il ne peut s'agir que d'un «modèle» théorique de référence regroupant en un ensemble logique les principes d'organisation spatiale propres à l'espace montagnard. Au point de départ, il y a l'énoncé d'un postulat: toutes les montagnes relèvent d'un même faisceau de causalités, elles sont organisées sur un même plan (pattern) et elles fonctionnent d'une manière identique. La montagne est une structure organisée par un "système » que l'on peut traiter comme un «champ spatial» (B. J. Berry) à partir d'un modèle théorique. Sur ce plan, le but est de sortir l'espace montagnard de l' "exceptionalisme » géographique (2).

Ce « modèle » montagnard n'est pour l'instant qu'une ébauche superficielle et partielle. Strictement "qualitatif», ce qui en limite la portée théorique et l'utilisation pratique, il ne regroupe que des faits depuis longtemps connus, soit des géographes, soit des naturalistes. Son élaboration passe par la sélection et le classement d'informations sectorielles disparates, géologiques, climatiques, hydrologiques, biogéographiques, anthropiques...). Le milieu montagnard n'est en fait qu'un cas particulier de paysage. On peut donc lui appliquer la méthode de traitement déjà élaborée et, en particulier, retenir comme fil directeur l'échelle spatiale des objets (3), ce qui permet d'établir une première classification parmi les structures écologiques (référence au système scalaire de CailleuxTricart) (4).

Cette esquisse ne peut donc être, dans un premier temps, que structurale et statique, c'est-à-dire topologique. Toutefois, l'échec déjà signalé des «définitions » strictement descriptives et structurales incite à se placer d'abord dans une perspective dynamique. Comme on l'a déjà montré (3), une unité de paysage se définit moins par une «limite » et une extension dans l'espace que par une autonomie de fonctionnement. La montagne doit d'abord être appréhendée comme un système spatial.

(2) F.K. Schaefer, Exceptionalism in geography. Annals of the Assoc. of American Geographers, 1953, vol. 43, p. 226249 et H. Capel, Exceptionalismo en geografia. Pensumiento y metódo geograficos. Depart. de geografía de la Universidad de Barcelona, 1971, $40 \mathrm{p}$.

(3) La science du paysage. Revue géographique des Pyrénées et du Sud-Ouest (Toulouse), 1972, n" 2.

14) J. Tricart, Principes et méthodes de la géomorphologie. Paris, Masson, 1965, p. 79-90.

\section{La montagne, "interface" turbulente.}

Comme toute portion d'épiderme terrestre, la montagne fonctionne comme unc «interfacc》 (5), c'est-à-dire comme une surface de contact le long de laquelle s'opèrent des transferts de matière et d'énergie entre les éléments de la lithosphère, de l'atmosphère, de l'hydrosphère et de la biosphère. C'est donc, par définition, une surface d'instabilité. Or, dans cette perspective dynamique, l'originalité de la montagne est d'être l' «interface» terrestre présentant le maximum de «rugosité», donc par voie de conséquence, le maximum d'instabilité, de «turbulence ».

En effet, la rugosité est d'abord liée à la protubérance même du volume montagneux global. Mais c'est aussi une rugosité interne, liée au compartimentage de la masse montagneuse, d'abord du point de vue topographique (juxtaposition massif-bassingorge, succession replat-paroi-plan incliné), mais aussi du point de vue des masses d'air, de la couverture végétale, etc. Pour une même surface projetée, l' «interface $\gg$ présente un plus grand développement en montagne qu'en plaine (parfois plus du double), la turbulence en est donc augmentée. De plus, $l$ ' «interface » montagnarde est très imbriquée et très contrastée, ce qui accroît encore les effets de turbulence: l'opposition entre une ombrée et une soulane en est l'illustration la plus classique. Cette «turbulence » (ou instabilité) généralisée de l'air, de l'eau, des manteaux dc débris, dc la couverture végétale, et même des compartiments tectoniques, constitue une sorte de loi écologique qui définit le milieu montagnard. Elle se vérifie à toutes les échelles (orogénèse d'un massif, effet de façade, effet topoclimatique, effet de catena, etc.). La montagne se définit donc comme un système traversé par un puissant flux énergétique qui se maintient à un niveau d'entropie minimum(6). De ce fait, l'espace montagnard se caractérise par un potentiel «morphologique * élevé, c'est-à-dire comme un milieu favorable à l'évolution (création, transformation, destruction) de «formes» au sens large (topographiques, climatiques, biologiques, etc.). Mais, avant d'appréhender le changement, il faut préciser la nature des structures plus stables qui organisent l'espace montagnard.

\section{Premiers éléments pour établir un "modèle topologique ".}

La montagne est, par définition, un fait topographique. C'est donc l'existence même du relief et son

(5) J. Tricart, La Terre, planète vivante. Paris, P.U.F., 1972.

(6) F. Bons, Vers une approche énergétique de l'environnement physique. Aspects théoriques. Bull. de recherche de l'Université de Sherbrooke (Québec), n" 7, janvier 1973, $74 \mathrm{p}$. 
TABLEAU 1. - Esquisse d'un «modèle écologique montagnard»

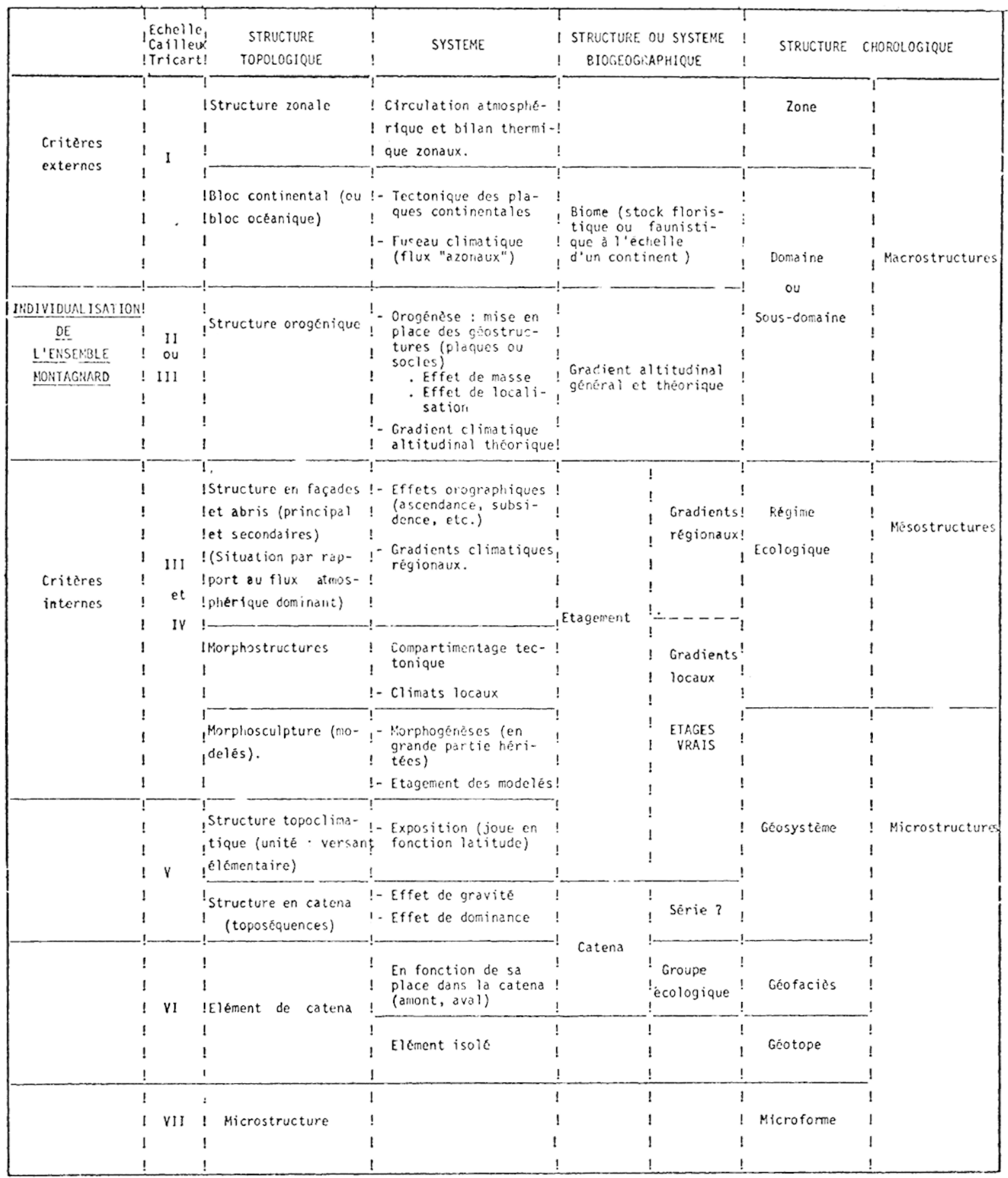

organisation volumétrique qui commandent la mosaïque écologique. Cette considération très banale a été pourtant négligée par la plupart des naturalistes, d'où le caractère théorique et laxiste de leurs divisions spatiales, tels les étages de végétation, qui ne tiennent, par exemple, aucun compte des faits géomorphologiques. On se propose d'esquisser une sorte de «grille» topologique de référence, caractérisant les grands niveaux d'organisation des espaces montagnards. En théorie, il s'agit de reconnaître des lois de distribution. Dans la pratique, il n'est pas encore possible de formuler de telles lois écologiques. On peut, au plus, les pressentir et les caractériser grossièrement sous la dénomination provisoire $d^{\prime}$ ' effets » écologiques.

a. Le volume rocheux global détermine l'effet de masse et le gradient écologique général.

Le volume montagneux lié à l'orogénèse donne l'échelle générale de tous les phénomènes, celle qui sert, en quelque sorte, d' « enveloppe » au «système » 
montagnard et agit sur les éléments qui se situent à des niveaux scalaires inférieurs (niveau II pour l'Himalaya, III pour les Pyrénées, IV pour les Vosges, etc.). Le volume rocheux se définit par ses composantes horizontales (localisation, extension, forme de l' «enveloppe ») et ses composantes verticales (élévation, distribution des altitudes). La combinaison de ces caractères topologiques conditionne directement les grandes structures écologiques :

- L'effet de masse. Il assure le degré d'individualisation (tectonique et écologique) de l'unité montagnarde par rapport aux unités voisines (continentales ou maritimes) et par rapport aux manifestations de la zonalité (degré de dépendance zonale).

- L'effet de localisation. La situation en latitude (effet zonal) se combine avec la position par rapport aux masses continentales et maritimes et avec l'orientation générale de la chaîne. A l'échelle des cordillères, on doit opposer les chaînes méridiennes plurizonales (Andes) aux chaînes "longitudinales» (est-ouest) monozonales (Himalaya). Le stock biologique (floristique, faunistique) dépend de l'ensemble de ces critères (problème des migrations, des «ponts », des isolats).

- L'effet de gradient général. Basé sur le gradient topographique, il se compose des gradients thermiques et pluviométriques et des divers gradients biogéographiques. Il commande l'étagement d'ensemble des paysages.

\section{b. L'effet de façade et d'abri corrélatif.}

Il dépend du volume montagneux (élévation), de la position en latitude et de l'orientation d'ensemble de la montagne par rapport au flux atmosphérique dominant (vent d'ouest, alizé, mousson, etc.). Un massif peut avoir une (Himalaya) ou deux façades (Andes). Ces dernières peuvent être plus ou moins actives, avec parfois des renversements saisonniers (façade principale, façade secondaire). La valeur des gradients, donc l'étagement, n'est pas identique sur une façade exposée à un flux atmosphérique actif et sur un versant abrité. C'est une première nuance apportée au principe d'étagement.

\section{c. Le compartimentage géomorphostructural.}

Ce compartimentage détermine (approximativement aux niveaux III et IV) la distribution des grandes unités topographiques (massifs, bassins et vallées) à l'intérieur de la montagne. Il résulte du jeu tectonique, mais il a pu être réaménagé par les glyptogénèses synorogéniques ou post-orogéniques. Dans les cordillères, les unités se disposent grossièrement en bandes parallèles plus ou moins comprimées ou dilatées (dans l'Himalaya: Siwaliks, Mahabharat Moyen Pays, etc.). Ce compartimentage a pour principales conséquences écologiques:

- l'apparition de discontinuités dans le gradient écologique général et la mise en place de gradients locaux avec des ruptures brutales, voire des inversions (mise en place des "étages» vrais).
- ces seuils déterminent le degré d'autonomie de chaque compartiment par rapport à l'ensemble montagneux. La taille et l'individualisation topographique du compartiment entrent donc en ligne de compte. Il existe par exemple un seuil isostatique (niveau III ?) au-dessous duquel un bloc montagneux pcrd la possibilité de réagir par compensation tectonique. Cette autonomie se retrouve, sous une autre forme, dans le domaine biogéographique (effet de masse, endémisme, écotypes, etc.).

\section{d. Les effets de fronts montagnards et d'abris cor- rélatifs.}

Ces effets (niveaux IV - V) sont la conséquence directe de la combinaison de l'effet de façade et du compartimentage géomorphostructural. En effet, chaque compartiment présente une hétérogénéité systématique qui est fonction de la direction et de l'activité du flux atmosphérique dominant. Du côté de la façade, chaque compartiment présente un front montagnard ( $H$. Gaussen), c'est-à-dire un versant soumis aux phénomènes d'ascendance orographique, donc plus humide et plus nébuleux que les autres secteurs de la montagne, en particulier que les versants «sous le vent». A mesure que l'on s'enfonce à l'intérieur de la masse montagneuse, l'effet de front s'atténue et l'optimum de précipitation et de nébulosité remonte sur le versant, alors que se renforce l'effet d'abri (effet d'écran d'un compartiment sur l'autre). L'effacement de l'effet de front peut devenir total et on peut avoir, dans la partie la plus abritée de la montagne, des mécanismes climatiques autonomes de bassin. Cette distribution systématique est beaucoup plus complexe quand il existe plusieurs effets de façades de puissance inégale et qui fonctionnent à des saisons différentes (Himalaya central).

\section{e. L'effet topoclimatique.}

C'est le rôle bien connu de l'exposition en montagne, fonction de la pente, modifié par la latitude et l'effet de masque. L'exposition au rayonnement solaire a été parfois confondu avec l'effet de façade ou de front montagnard. En fait, c'est la combinaison entre ces deux mécanismes, indépendants et jouant à des échelles spatiales différentes, qui permet d'expliquer un nombre important de faits bioclimatiques et de situations écologiques. Par exemple, une soulane de front montagnard externe se différencie d'une soulane de front montagnard interne; une ombrée de façade d'une ombrée de versant «sous le vent». C'est un moyen de classer les géosystèmes montagnards.

\section{f. Les catenas.}

A l'échelle du versant élémentaire, c'est-à-dire du plan plus ou moins incliné non interrompu par un écoulement transversal, ce sont les phénomènes de gravité qui organisent en priorité l'espace montagnard (circulation de l'eau, de l'air, des débris) à partir d'une dynamique essentiellement «oblique». 
La plupart des géofaciès et des géotopes se caractérisent par leur position (amont, aval...) dans la chaîne de versant.

\section{Du " modèle" statique au " modèle " dynami- que.}

Ce schéma topologique gigogne reste entièrement statique. Il essaie de retrouver et de codifier la logique de l'organisation spatiale en montagne. Mais il manque encore d'information et de cohérence. Toutefois, il permet d'ébaucher une première classification comparative des unités de paysage. Chaque unité d'un même système taxonomique (par exemple : région naturelle, géosystème, géofaciès, géotope) est «mise en situation», à son échelle spatiale, en fonction, d'une part, de l'ensemble écologique fonctionnel auquel elle se rattache (façade, front montagnard, soulane, etc.), d'autre part, des ensembles écologiques qu'elle contient.

a. L'instabilité structurelle est, plus encore que dans les autres espaces écologiques, la condition de l'existence, donc de l'équilibre, des milieux montagnards. L'instabilité est «climacique 》 (au sens étymologique), d'où l'inadéquation en montagne du concept de climax dans son acception classique, et surtout des présupposés cycliques qui l'accompagnent habituellement (le « retour au climax» est, en fait, à la base de la conception de série régressive ou progressive de végétation). Le déterminisme écologique stationnel à l'état brut s'efface devant la probabilité, qui incorpore le temps. Comme on a pu le montrer dans les Montagnes cantabriques centrales, deux soulanes identiques du point de vue du «substrat» géomorphologique et bioclimatique, mais dans des positions géographiques différentes par rapport aux « réservoirs » floristiques, l'une dans un grand bassin, l'autre dans une petite vallée latérale et isolée, peuvent avoir, à un moment donné, des formations végétales et des sols différents.

b. Les interrelations dans le temps et dans l'espace. Elles sont, a priori, à l'origine même de la dynamique des milieux montagnards. Dans l'espace, la finesse et les contrastes de la mosaïque écologique rendent les interactions entre milieux, ou éléments de milieux, fréquentes et souvent déterminantes. C'est le principe même de l'effet de catena. De façon plus générale, il faut souligner le rôle essentiel de l'effet de dominance. Lié à la gravité, il est, du fait de la rugosité de l'interface, spécifique des milieux montagnards. Ces phénomènes de dominance sont très connus: vents catabatiques et "coulées froides》, éboulisation, stations végétales «abyssales 》 (H. Gaussen), etc. Dans le temps, la fréquence du franchissement de certains seuils géologiques ou écologiques fondamentaux (seuil isostatique, pente d'équilibre d'un versant, seuil mécanique et biochimique, seuil du 0 " $C$, seuil léthal), et des interactions entre l'ensemble de ces ruptures, multiplie sous nos yeux les cas de dé- phasages et d'hystérésis. L'intervention des activités agro-sylvo-pastorales, et aussi touristiques, accentue encore cette tendance à la diversification spatiale et à la «turbulence» temporelle. L'idée du «catastrophisme » montagnard n'est pas une vue de l'esprit. Il devrait être reconsidéré dans cette optique à la fois spatiale et dynamique.

c. Les milieux montagnards présentent un état remarquable d' «hétérostasie» (H. Erhart). C'est encore un fait banal d'observation: une soulane surpâturée, décapée, ravinée, peut voisiner avec une ombrée colonisée par une haute futaie longuement mûrie sur place. Certains écologistes y voient une justification de la notion de «polyclimax». En fait, la distribution des climax, même théoriques, n'est pas stable dans l'espace: l'ombrée peut aussi être dégradée dans certaines conditions bioclimatiques et géomorphogéniques. Des ensembles écologiques voisins peuvent donc être l'objet d'évolution différentes, voire contradictoires. En montagne plus qu'ailleurs, l'espace et le temps interfèrent dans une dialectique qui n'est pas encore bien débrouillée. Cela amène plusieurs remarques :

- on ne peut pas encore élaborer un «modèle» montagnard dynamique; pour le moment, la seule méthode acceptable consiste à établir le modèle statique actuel (cf. Himalaya); puis, par la méthode historique "régressive », à reconstituer un certain nombre de «modèles» caractéristiques (par exemple, le modèle du Maximum glaciaire quaternaire);

- les tentatives de chronologies sectorielles (phases glaciaires ou périglaciaires, stades phytocinétiques) qui tirent des conclusions générales de l'examen de phénomènes isolés de leur contexte écologique doivent, a priori, être considérées avec beaucoup de réserve; les reconstitutions historiques ne tiennent pas assez compte de l'hétérogénéité structurelle des milieux de montagne; le passage des images stationnelles (fournies par l'étude de «coupes» ou l'analyse des pollens d'une tourbière) à la généralisation devrait s'appuyer sur un examen approfondi des structures et des dynamiques spatiales actuelles ou subactuelles;

- de ce fait, il faut considérer comme une erreur méthodologique grave de privilégier les reconstitutions chronologiques par rapport à l'observation systématique de la distribution actuelle des phénomènes; l'expérience de recherches conduites dans les Montagnes cantabriques montre qu'une bonne connaissance du modèle écologique actuel, et tout spécialement de son hétérogénéité, permet de mieux comprendre certaines évolutions et, en particulier, de limiter la portée des grandes hypothèses classiques (fluctuations climatiques du Quaternaire, vastes courants migratoires pour les plantes et les animaux) (3);

- en somme, la vieille méthode "naturaliste», parfois critiquée par les tenants de la «nouvelle géographie », qui part de l'observation et de la classification des faits actuels, et qui progresse par contrôle historique "régressif 》, s'avère bien supérieure à la méthode historique héritée des géologues, puis des 
géomorphologues, pour lesquels le souci de datation, c'est-à-dire le temps, l'emporte souvent sur l'espace, c'est-à-dire sur la distribution actuelle des phénomènes.

D'une définition brève et contestable de l'espace écologique montagnard, on est parvenu à la conception d'un modèle théorique et qualitatif. $\mathrm{Ce}$ dernier revêt d'abord un aspect structural et statique, qu'il est possible d'élaborer même si les lois de dis- tribution se dégagent encore assez mal du magma des interférences. Par contre, la construction d'un modèle dynamique paraît encore impossible. Toutefois, on peut dès à présent en fixer les principes généraux et surtout révéler un certain nombre de points d'achoppement. Il semble que l'on soit arrivé à un seuil de la réflexion et de la méthode à partir duquel le raisonnement «qualitatif» doit être transcendé par l'analyse mathématique, seule susceptible d'élaborer un modèle exhaustif fiable, pratique.

La rédaction de L'Espace géographique recevra volontiers les questions, critiques, observations ou suggestions que susciterait cette publication.

Adresser la correspondance à l'un des deux directeurs, ou à la rédaction de L'Espace géographique. 